\title{
ANTIBIOTIC RESISTANCE PATTERN OF PSEUDOMONAS AERUGINOSA ISOLATED FROM VARIOUS CLINICAL SAMPLES IN A TERTIARY CARE HOSPITAL, PUDUCHERRY
}

\author{
KANTHAKUMAR A, JAYAVARTHINNI M* \\ Department of Microbiology, Indira Gandhi Medical College and Research Institute, Puducherry, India. Email: jayavarthinni@yahoo.co.in \\ Received: 02 December 2021, Revised and Accepted: 05 January 2022
}

\begin{abstract}
Objective: One of the most common bacteria known to cause nosocomial infection and found to be multidrug-resistant is Pseudomonas aeruginosa. The objective of the study was to know the prevalence of the $P$. aeruginosa isolates with varied clinical conditions and specimens and to assess the antimicrobial susceptibility patterns of P. aeruginosa as well as its magnitude of multidrug resistance (MDR).
\end{abstract}

Methods: A total of 229 biochemically tested and confirmed isolates of $P$. aeruginosa from various clinical samples were studied. Antibiotic susceptibility testing was determined by Kirby-Bauer disc diffusion method.

Results: Out of the 229 isolates of $P$. aeruginosa, majority (60.70\%) were from pus sample. Resistance to amikacin and tobramycin was $23.6 \%$ and $20.1 \%$, ciprofloxacin was $33.2 \%$. Resistance to ceftazidime, cefoperazone and cefepime were $21.8 \%$, $45.9 \%$, and $25.7 \%$. Imipenem and meropenem showed $26.2 \%$ and $20.5 \%$ resistance, respectively. Resistance to piperacillin was $18.3 \%$ while piperacillin-tazobactam was only $13.5 \%$. The MDR was observed in $33.7 \%$ of the isolates.

Conclusion: There is increased resistance to cephalosporins as compared to aminoglycosides, carbapenems and beta lactamase inhibitor. To restrict the inappropriate use of antimicrobial agents, the development of MDR, needs to be continuously monitored and documented.

Keywords: Multidrug resistance, Pseudomonas aeruginosa, Antimicrobial susceptibility pattern.

(C) 2022 The Authors. Published by Innovare Academic Sciences Pvt Ltd. This is an open access article under the CC BY license (http://creativecommons.org/ licenses/by/4.0/) DOI: http://dx.doi.org/10.22159/ajpcr.2022v15i2.43839. Journal homepage: https://innovareacademics.in/journals/index.php/ajpcr

\section{INTRODUCTION}

Pseudomonas aeruginosa is the bacterium that is known to cause persistent infections for prolonged duration that could end up in serious outcomes in terms of morbidity and mortality. The introduction of a variety of sanitation facilities and antimicrobial agents with antipseudomonal activities has not reduced the life-threatening hospital based infections caused by it [1]. P. aeruginosa is inherently resistant to many antimicrobial agents, thus posing a great challenge in community acquired and nosocomial infections [2]. The occurrence of multidrug resistance (MDR) in these isolates is of serious concern as it poses a problem in therapy and infection control management $[3,4]$. The prevalence and antimicrobial susceptibility pattern of the resistant isolates in different geographical settings by epidemiological studies would give useful information to add to the global picture of antimicrobial resistance thereby guiding the clinicians in their choice of antimicrobial therapy [1]. In view of these facts, the present study was undertaken to find out the prevalence of multi drug resistance and antibiotic susceptibility patterns of pathogenic $P$. aeruginosa isolated from various clinical specimens in a tertiary care hospital.

\section{METHODS}

The study was conducted in the Microbiology Department of Indira Gandhi Medical College and Research Institute, Puducherry, after obtaining the Institute Ethics Committee approval. A record based retrospective analysis of data of all samples received over a 1 year period from January 2017 to December 2017 yielding a growth of $P$. aeruginosa was under taken. Identical report from the same patient was excluded from the study.

The isolates were identified by conventional methods. The strains were identified as P. aeruginosa, based on the colony morphology, gram staining, oxidase reaction, the production of the pyocyanin pigment, fruity odor, nitrate reduction, the use of citrate and malonate as carbon sources, and its ability to grow at $42^{\circ} \mathrm{C}$ [5]. P. aeruginosa ATCC 27853 was used as control.

Antibiotic susceptibility for $P$. aeruginosa was performed using the Kirby-Bauer disk diffusion method according to Clinical and Laboratory Standards Institute guidelines using commercially available disks (HiMedia, Mumbai): amikacin (30 $\mu \mathrm{g}$ ), ciprofloxacin (5 $\mu \mathrm{g})$, ceftazidime (30 $\mu \mathrm{g})$, cefoperazone $(75 \mu \mathrm{g})$, piperacillin $(100 \mu \mathrm{g})$, tobramycin (10 $\mu \mathrm{g})$, cefepime $(30 \mu \mathrm{g})$, imipenem $(10 \mu \mathrm{g})$, meropenem $(10 \mu \mathrm{g})$, and piperacillin-tazobactam $(100 / 10 \mu \mathrm{g})$ [6]. Isolates with intermediate levels of resistance in disk diffusion were included in the percentage of resistant organisms for final analysis. Isolates were considered multidrug resistant (MDR) if they showed resistance to three or more classes of the tested antibiotics [7]. All the data were entered and analyzed in Microsoft Excel 2010 and expressed as percentages.

\section{RESULTS}

A total of 229 P. aeruginosa isolates from various clinical samples comprised of 120 (52.4\%) males and 109 (47.6\%) females, with a male: female ratio of 1.1:1. The age of the patients ranged between 1 month and 105 years, with a median of 45 years. Age-wise and sexwise distribution of $P$. aeruginosa isolates is shown in Table 1.

Out of the 229 isolates of P. aeruginosa, 139 (60.70\%) were from pus, $33(14.41 \%)$ were from sputum, 27 (11.79\%) were from urine, $16(6.99 \%)$ were from blood, and 14 (6.11\%) were from other samples. The distribution of $P$. aeruginosa among various clinical samples is shown in Fig. 1.

The antimicrobial resistance pattern of $P$. aeruginosa isolates is shown in Table 2. Resistance to amikacin and tobramycin were $23.6 \%$ and $20.1 \%$, ciprofloxacin was $33.2 \%$. Resistance to ceftazidime, cefoperazone and 
cefepime were $21.8 \%, 45.9 \%$, and $25.7 \%$. Imipenem and meropenem showed $26.2 \%$ and $20.5 \%$ resistance, respectively. Resistance to piperacillin was $18.3 \%$ while piperacillin-tazobactam was only $13.5 \%$. The MDR was observed in $33.7 \%$ of the isolates.

\section{DISCUSSION}

The ubiquitous Gram-negative bacterium belonging to the family Pseudomonadaceae, P. aeruginosa, has the ability to survive in a wide range of environments and thereby complicating the therapeutic approaches for treatment. In the present study, a total of $229 P$. aeruginosa isolates were isolated from various clinical samples. There was no difference in sex-wise distribution, with a male: female ratio of 1.1:1. This was concordance to the study conducted by Dash et al. [8] who reported the male: female ratio as 1.4:1. Both men and women were equally affected with $P$. aeruginosa infection. The predominant age group distribution was between 19 and 50 years (50.6\%) followed by the elderly age group $>50$ years $(36.7 \%)$. This was similar to the study reported by Chander et al. [7] in which most of them belonged to older age group of 21-40 years (41.4\%) and elderly age group of $>60$ years (31\%). The factors such as low-level of immunity, prolonged hospitalization and other associated comorbidities could be the possible reasons for the elderly people being affected.

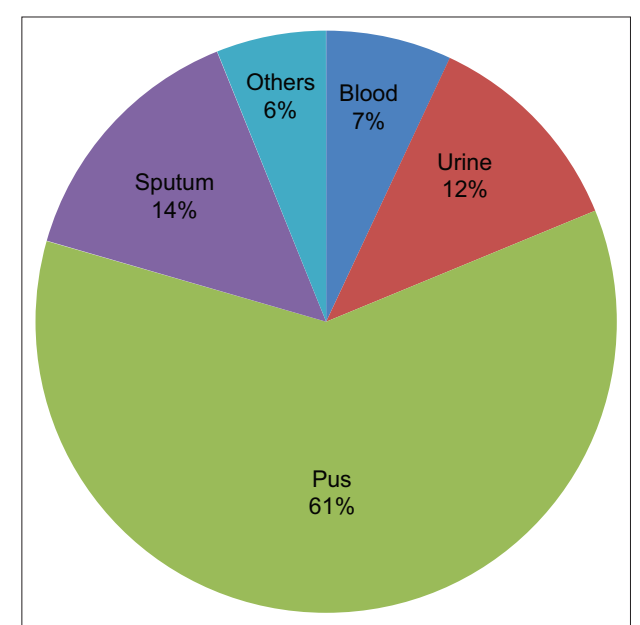

Fig. 1: Distribution of Pseudomonas aeruginosa among various clinical isolates

Table 1: Age-wise and sex-wise distribution of Pseudomonas aeruginosa isolates

\begin{tabular}{llll}
\hline Age (years) & No. of Male (\%) & No. of Female (\%) & Total (\%) \\
\hline $0-18$ & 18 & 11 & $29(12.7)$ \\
$19-50$ & 53 & 63 & $116(50.6)$ \\
$>50$ & 49 & 35 & $84(36.7)$ \\
Total & $120(52.4)$ & $109(47.6)$ & $229(100)$ \\
\hline
\end{tabular}

Table 2: Resistance pattern of Pseudomonas aeruginosa isolates

\begin{tabular}{ll}
\hline Antibiotics & $\begin{array}{l}\text { Pseudomonas aeruginosa } \mathbf{n}=\mathbf{2 2 9} \text { (\% of } \\
\text { resistance) }\end{array}$ \\
\hline Amikacin & $54(23.6)$ \\
Ciprofloxacin & $76(33.2)$ \\
Ceftazidime & $50(21.8)$ \\
Cefoperazone & $105(45.9)$ \\
Piperacillin & $42(18.3)$ \\
Tobramycin & $46(20.1)$ \\
Cefepime & $59(25.7)$ \\
Imipenem & $60(26.2)$ \\
Meropenem & $47(20.5)$ \\
Piperacillin-tazobactam & $31(13.5)$ \\
\hline
\end{tabular}

Out of the 229 isolates of P. aeruginosa, $60.70 \%$ were from pus sample, followed by sputum (14.41\%), urine $(11.79 \%)$, blood (6.99\%), and other samples $(6.11 \%)$. This defines the fact that it is commonly isolated from wound infection. This was concordance to the other studies conducted from different parts of India $[1,2,9]$.

The resistance pattern of $P$. aeruginosa isolates was evaluated with ten different antimicrobial agents. The fluoroquinolone, ciprofloxacin showed $33.2 \%$ resistance. This was partly concordance to the studies conducted by Hoque et al. [10], Chander et al. [7] and Kaur et al. [2] who reported the resistance as $54 \%, 51.72 \%$, and $44.2 \%$, respectively.

Among the aminoglycosides, amikacin and tobramycin showed $23.6 \%$ and $20.1 \%$ of resistance. Tiwari et al. [11] reported higher level of resistance to amikacin and tobramycin as $41 \%$ and $39 \%$ compared to our study. However, resistance to amikacin varied from as low as $13.3 \%$ and as high as $81 \%$ in other studies $[10,12]$. This could be due to choice of the antimicrobials used in their health setup.

In our study, the carbapenems, imipenem, and meropenem exhibited $26.2 \%$ and $20.5 \%$ of resistance. This was partly concordance to the study conducted by Kaur et al. [2] who reported the resistance as $17.8 \%$ and $33.1 \%$, respectively. In contrast, Kumari et al. [13] reported the resistance as $53 \%$ and $63 \%$ for imipenem and meropenem, respectively. As carbapenems remain the main stay of antimicrobial of choice especially to MDR P. aeruginosa, the lower level of resistance to them is eventually essential. Even a lower percentage of resistance is quite mindful.

Among the beta lactams, the third generation cephalosporins, ceftazidime showed $21.8 \%$ of resistance while cefoperazone showed high level of resistance $(45.9 \%)$, which may be contributed to the fact that it is the commonly used antimicrobial agent. In a study conducted by Rustini et al. [14], ceftazidime showed $26.32 \%$ of resistance while cefoperazone showed $39.89 \%$ of resistance on par with our study. In our study, the fourth generation cephalosporin namely cefepime exhibited $25.7 \%$ of resistance. Very low level of resistance was reported by Saroj et al. [12] to ceftazidime (8.92\%) and cefepime $(4.46 \%)$ and high level of resistance was reported by Kaur et al. [2] as 62.8\% to ceftazidime and $61.1 \%$ to cefepime. Similarly, Mohanasoundaram [1] reported higher level of resistance for ceftazidime (63.3\%) and cefepime (72.3\%). The commonly used cephalosporins develop higher level of resistance rapidly and it is evident that judicious use of the cephalosporins is mandated to prevent the development of resistance.

The beta lactam agent, piperacillin showed $18.3 \%$ of resistance. However, piperacillin-tazobactam (beta lactamase inhibitor agent) showed only $13.5 \%$ of resistance similar to the study conducted by Tiwari et al. [11] who reported $32 \%$ of resistance to piperacillin and $26 \%$ of resistance to piperacillin-tazobactam. As evidenced by the study conducted by Choudhary et al. [3] the metallo beta lactamase producing $P$. aeruginosa were found to be $80.55 \%$ resistant to piperacillintazobactam compared to non metallo beta lactamase producing P. aeruginosa which showed $38.88 \%$ of resistance to piperacillintazobactam [3]. This signals the importance of MDR.

The prevalence of MDR, i.e., resistance to more than three classes of antimicrobial agents, among P. aeruginosa isolates were $33.6 \%$ $(77 / 229)$. This was concordance to the study conducted by Pramodhini et al. [15] and Saroj et al. [12] in which the MDR rate was 25\% and $8.92 \%$, respectively, but in contrast to the study reported by Mohanasoundaram [1], the MDR rate was $71 \%$. This highlights the fact that the resistance rate needs a constant check.

It is welcoming that the aminoglycosides (amikacin and tobramycin), carbapenems (imipenem and meropenem), and beta lactamase inhibitor (piperacillin-tazobactam) have shown lower level of resistance $(<26 \%)$ to $P$. aeruginosa isolates, but the resistance exhibited by cephalosporins in specific cefoperazone is $45.9 \%$, is quite alarming. 


\section{CONCLUSION}

The limited susceptibility to antimicrobial agents and high frequency of emergence of antibiotic resistance during therapy has complicated the treatment of $P$. aeruginosa infections. Therefore, it is of prime importance to continuously monitor the development of drug resistance in this group of organisms. Furthermore, judicious use of antimicrobial agents is the need of the hour to combat the development of antimicrobial resistance.

\section{AUTHORS' CONTRIBUTIONS:}

Mr. Kanthakumar. A was involved in the planning and execution of the study. Dr. Jayavarthinni. M was involved in writing, reviewing of the manuscript and also in execution of the study.

\section{CONFLICT OF INTEREST}

The authors have no known conflicts of interest to declare.

\section{AUTHORS' FUNDING}

No external funds were received for this research.

\section{REFERENCES}

1. Mohanasoundaram KM. The antibiotic resistance pattern in the clinical isolates of Pseudomonas aeruginosa in a tertiary care hospital; 20082010 (A 3 year study). J Clin Diagn Res 2011;5:491-4.

2. Kaur A, Singh S, Gill AK, Kaur N. Prevalence and antimicrobial susceptibility pattern of Pseudomonas aeruginosa isolated from various clinical samples in tertiary care Hospital, Bathinda. Indian J Basic Appl Med Res 2016;5:777-84.

3. Choudhary V, Pal N, Hooja S. Prevalence and antibiotic resistance pattern of Metallo- $\beta$-lactamase-producing Pseudomonas aeruginosa isolates from clinical specimens in a tertiary care hospital. J Mahatma Gandhi Inst Med Sci 2019;24:19-22.

4. Horcajada JP, Montero M, OliverA, Sorlí L, Luque S, Gómez-Zorrilla S, et al. Epidemiology and treatment of multidrug-resistant and extensively drug-resistant Pseudomonas aeruginosa infections. Clin Microbiol Rev 2019;32:e19-31.

5. Collee JG, Miles RS, Watt B. Tests for identification of bacteria. In: Collee JG, Fraser AG, Marmion BP, Simmons A, editors. Mackie and McCartney Practical Medical Microbiology. $14^{\text {th }}$ ed. Singapore: Churchill Livingstone; 2006. p. 131-49.

6. Clinical and Laboratory Standards Institute. Performance Standards for Antimicrobial Susceptibility Testing; Twenty Seventh Informational Supplement M100. Wayne, PA: Clinical and Laboratory Standards Institute; 2017.

7. Chander A, Raza MS. Antimicrobial susceptibility patterns of Pseudomonas aeruginosa clinical isolates at a tertiary care hospital in Kathmandu, Nepal. Asian J Pharm Clin Res 2013;6:235-8.

8. Dash M, Padhi S, Narasimham MV, Pattnaik S. Antimicrobial resistance pattern of Pseudomonas aeruginosa isolated from various clinical samples in a tertiary care hospital, South Odisha, India. Saudi J Health Sci 2014;3:15-9.

9. Yadav S, Sujatha R, Deepak S. Emergence of multidrug resistant Pseudomonas aeruginosa in a tertiary care center. Int J Health Sci Res 2018;8:52-5.

10. Hoque MM, Ahmad M, Khisa S, Uddin MN, Jesmine R. Antibiotic resistance pattern in Pseudomonas aeruginosa isolated from different clinical specimens. J Armed Forces Med Coll Bangladesh 2015;11:45-9.

11. Tiwari N, Rajdev S, Mullan S. Resistance trends among Pseudomonas aeruginosa isolates in a tertiary care centre in South Gujarat. Adv Microbiol 2017;7:188-94.

12. Saroj G, Suhani, Manasa S, Jyoti. Isolation of Pseudomonas aeruginosa from various clinical isolates and it antimicrobial resistance pattern in a tertiary care hospital. Int J Curr Microbiol Appl Sci 2016;5:247-53.

13. Kumari M, Khurana S, Bhardwaj N, Malhotra R, Mathur P. Pathogen burden and associated antibiogram of Pseudomonas spp in a tertiary care hospital of India. Indian J Med Res 2019;149:295.

14. Rustini R, Jamsari J, Marlina M, Zubir N, Yuliandra Y. Antibacterial resistance pattern of Pseudomonas aeruginosa isolated from clinical samples at a general hospital in Padang, West Sumatra, Indonesia. Asian J Pharm Clin Res 2017;10:158-60.

15. Pramodhini S, Umadevi S, Seetha KS. Prevalence of Antimicrobial resistance in clinical isolates of Pseudomonas aeruginosa in a tertiary care hospital, Puducherry, India. Int J Curr Microbiol Appl Sci 2015;4:718-26. 